\title{
Inactivation of DNase1L2 and DNase2 in keratinocytes suppresses DNA degradation during epidermal cornification and results in constitutive parakeratosis
}

\section{Heinz Fischer, Maria Buchberger, Erwin Tschachler, Leopold Eckhart}

Research Division of Biology and Pathobiology of the Skin, Department of Dermatology, Medical University of Vienna, Austria

\begin{abstract}
Nuclear DNA of keratinocytes is degraded during the formation of the stratum corneum. Here, we investigated the mechanism of cornificationassociated DNA degradation by generating mouse models deficient of candidate DNA-degrading enzymes and characterizing their epidermal phenotypes. In contrast to DNase1L2 knockout mice and keratinocytespecific DNase2 knockout mice, DNase1L2/DNase2 double knockout mice aberrantly retained nuclear DNA in the stratum corneum, a phenomenon commonly referred to as parakeratosis. The DNA within DNase1 L2/DNase2-deficient corneocytes was partially degraded, indicating that an additional, though insufficient mechanism of DNA fragmentation operates beside DNase1L2 and DNase2. Isolation of corneocytes and in situ DNA labelling demonstrated that corneocytes of DNase1 L2/DNase2 double knockout mice contained DNA in a nucleus-like compartment that also contained nucleosomal histones but lacked the nuclear intermediate filament protein lamin A/C. Parakeratosis was not associated with altered corneocyte resistance to mechanical stress, changes in transepidermal water loss, or inflammatory infiltrates in DNase1L2/DNase2 double knockout mice. The results of this study suggest that cornification of epidermal keratinocytes depends on the cooperation of DNase1L2 and DNase2 and indicate that parakeratosis per se does not suffice to cause skin pathologies.
\end{abstract}

\section{Generation of DNase knockout mice}

DNase1L2 knockout: Fischer et al., JID 2011 parakeratosis in hair and nails

DNase2 knockout: Fischer et al., JID 2017

DNase $2^{\mathrm{f} / \mathrm{f}} \mathrm{K} 14$-Cre, deletion in $\mathrm{K} 14$ epithelia

- suppression of DNA degradation in sebaceous glands

Double knockout:

DNase1L2/DNase2 DKO by crossing single-KO mice

\section{Results}

Double knockout of DNase1L2 and DNase2 causes parakeratosis: retention of DNA and histone $\mathrm{H}_{3}$

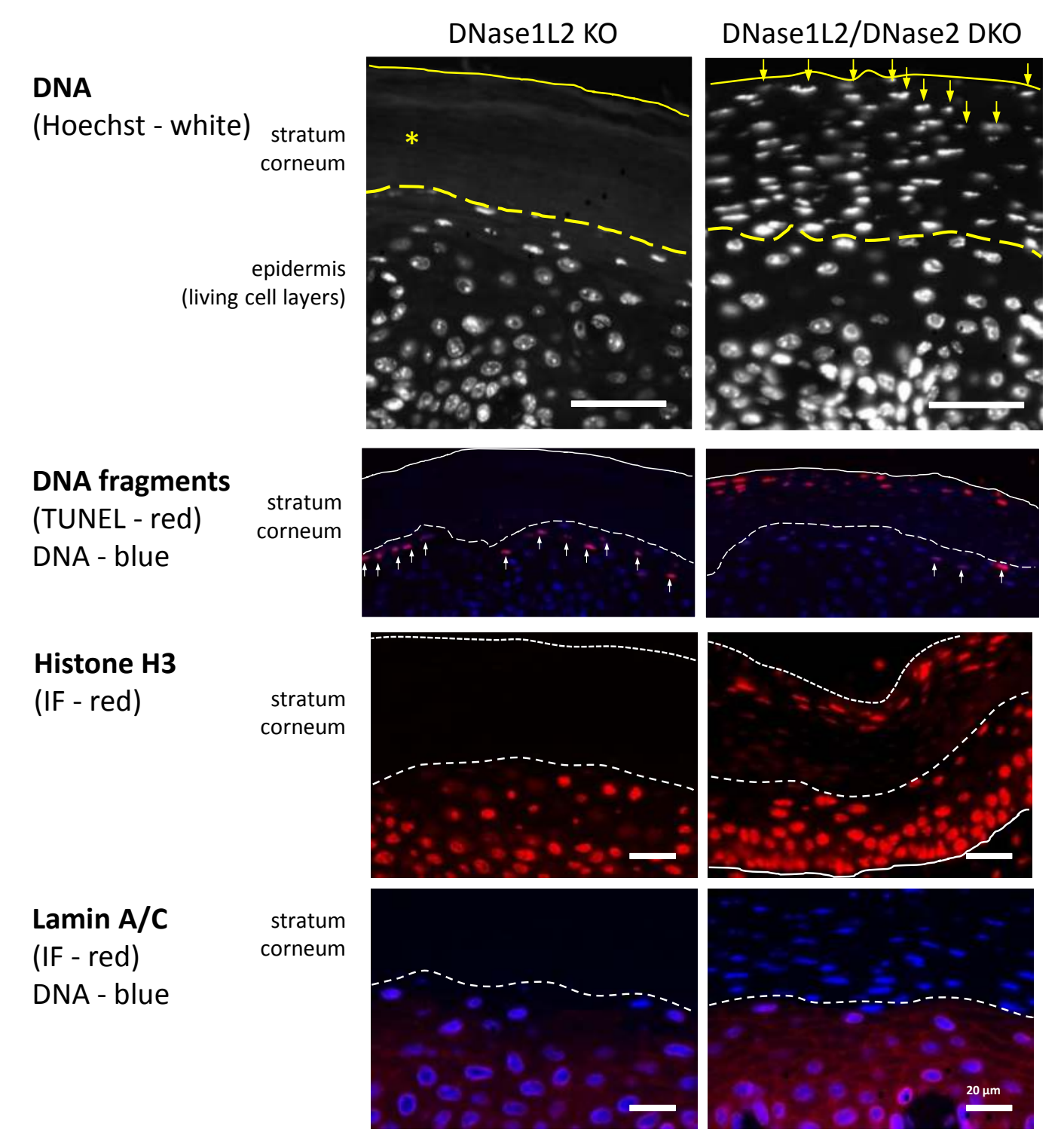

Thin section through the sole skin of DNase1L2 KO mice and Dnase1L2/Dnase2 double knockout (DKO) mice were labeled for DNA, TUNEL, histone H3 and lamin A/C. Results for wildtype and DNase2 KO mice (not shown) were similar to those of DNase IL2 KO mice, that is, normal orthokeratosis.
Tape-stripped corneocytes of DNase1L2/DNase2 double knockout mice contain DNA

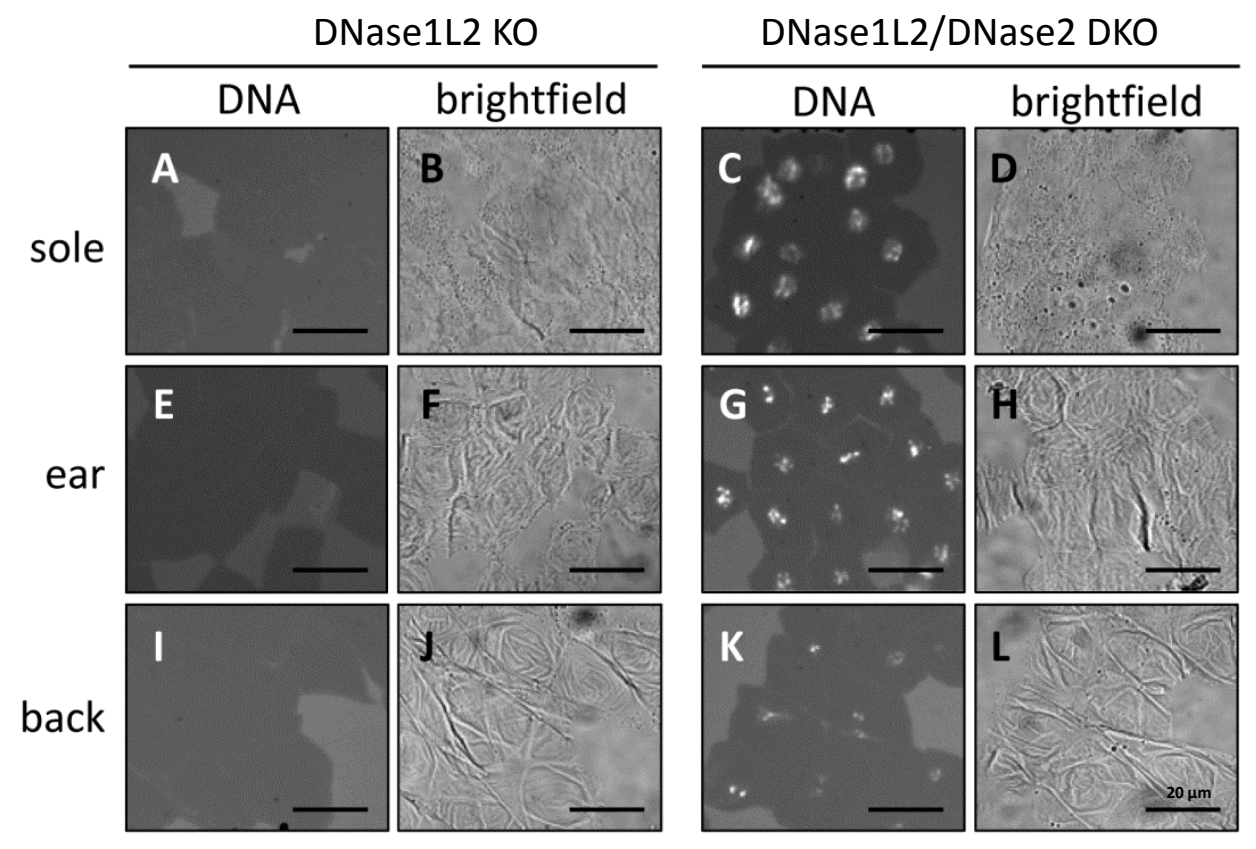

Corneocytes of DNase1L2/DNase2 are stress-resistant

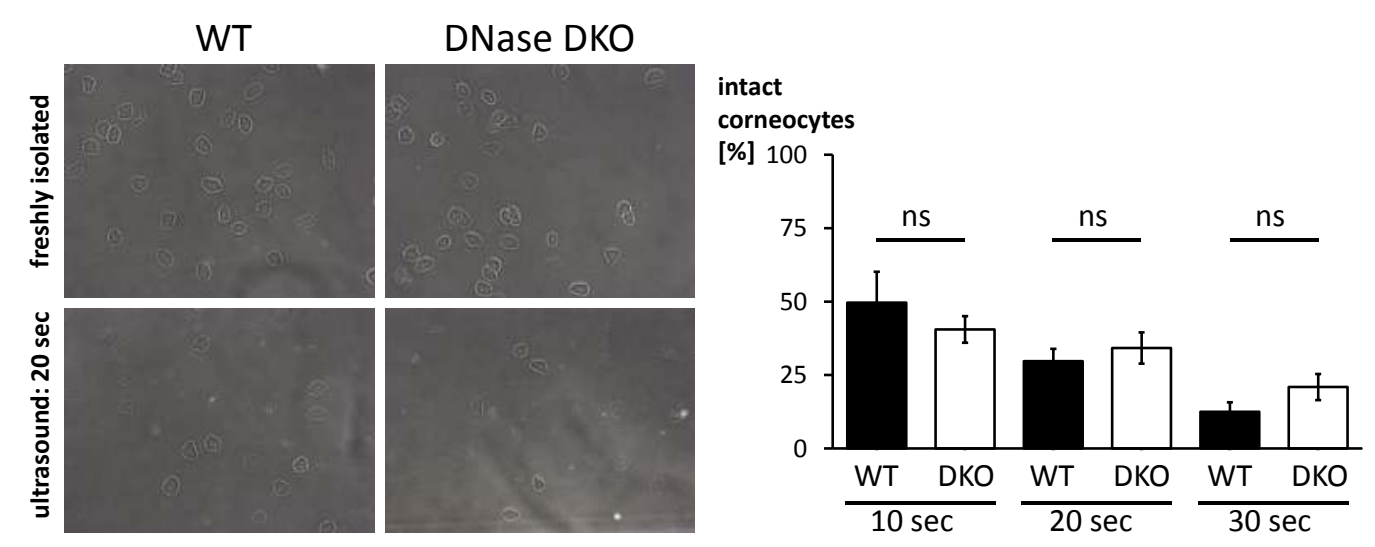

DNase knockout does not lead to skin inflammation

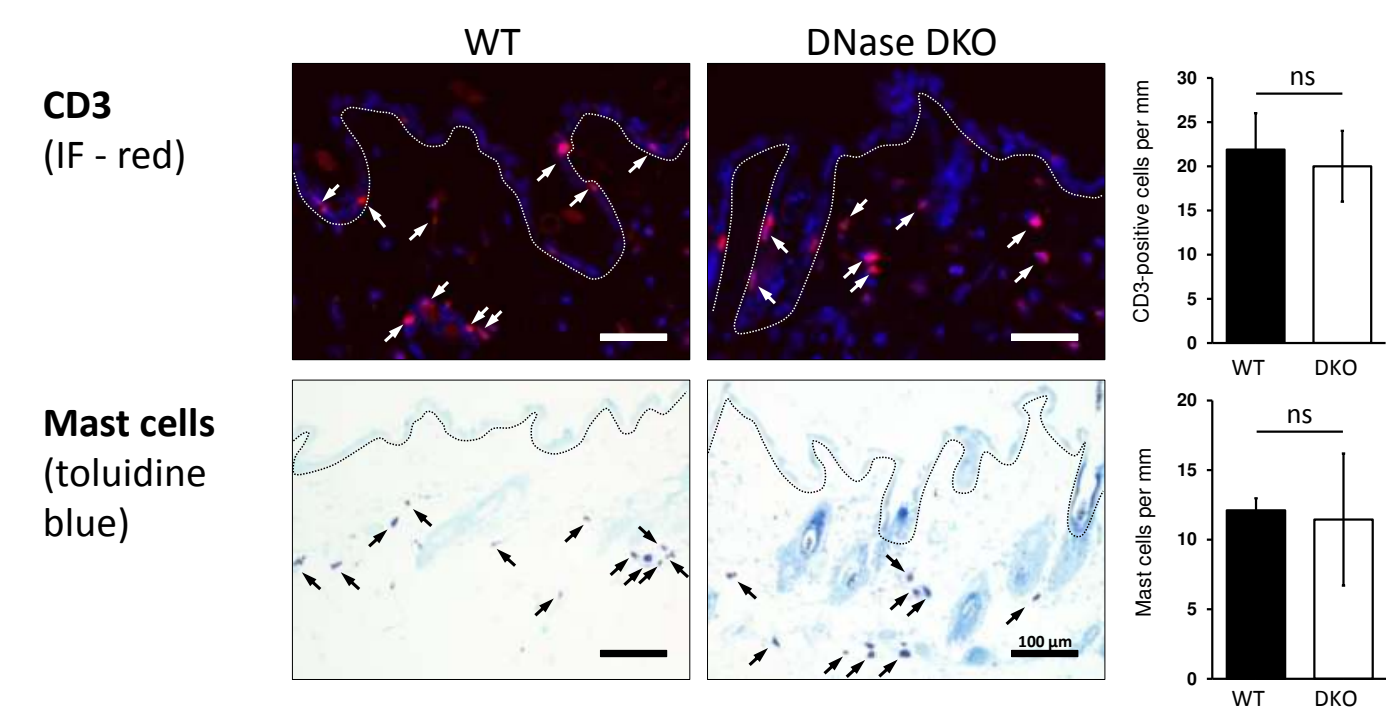

Summary and conclusions

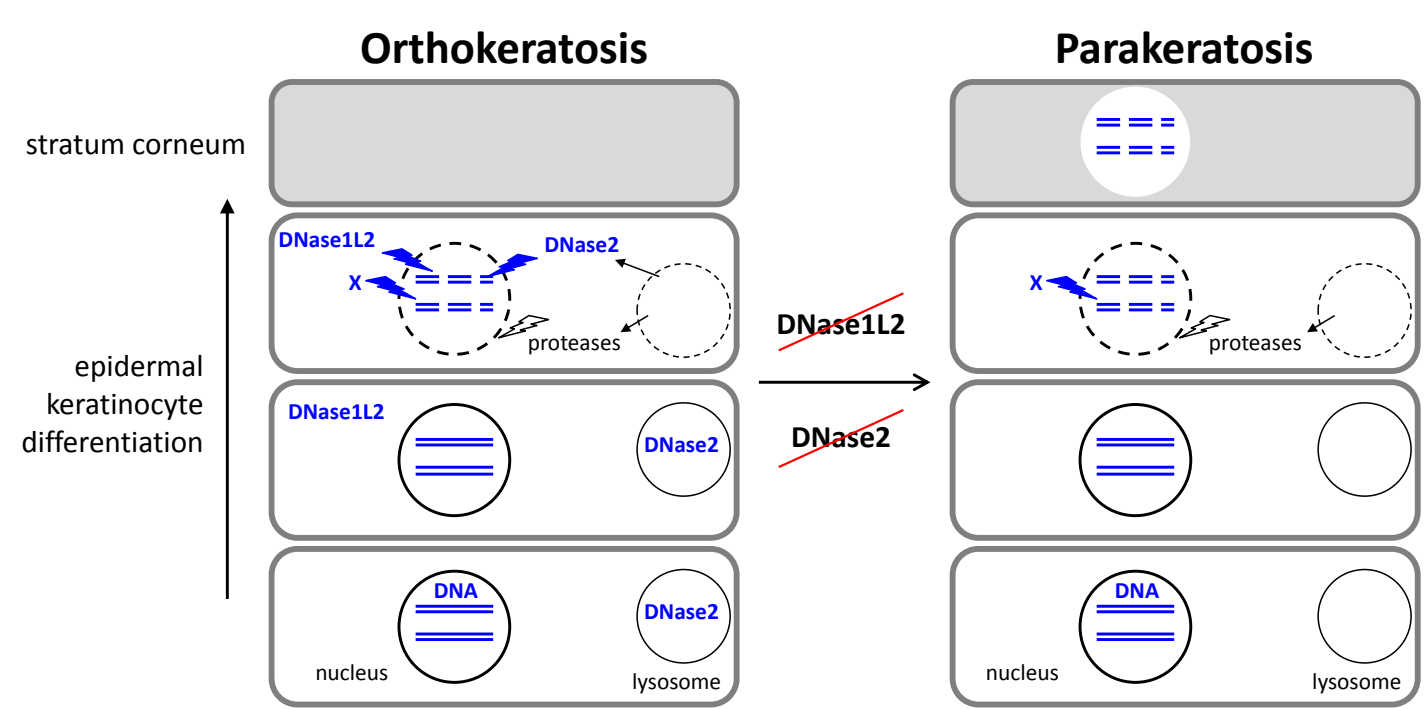

- DNase1L2 and DNase2 cooperate in DNA degradation during cornification.

- The contribution of DNase2 suggests a role of lysosomes in this process.

- DNA retention in cornifying keratinocytes does not cause skin inflammation. 